\title{
DIRAC STRUCTURES FROM LIE INTEGRABILITY
}

\author{
MIRCEA CRASMAREANU \\ Faculty of Mathematics \\ University "Al. I. Cuza" \\ Iaşi 700506, România \\ mcrasm@uaic.ro \\ Received 21 June 2011 \\ Accepted 9 November 2011 \\ Published 4 April 2012
}

\begin{abstract}
We prove that a pair ( $F=$ vector sub-bundle of $T M$, its annihilator) yields an almost Dirac structure which is Dirac if and only if $F$ is Lie integrable. Then a flat Ehresmann connection on a fiber bundle $\xi$ yields two complementary, but not orthogonally, Dirac structures on the total space $M$ of $\xi$. These Dirac structures are also Lagrangian subbundles with respect to the natural almost symplectic structure of the big tangent bundle of $M$. The tangent bundle in Riemannian geometry is discussed as particular case and the 3-dimensional Heisenberg space is illustrated as example. More generally, we study the Bianchi-Cartan-Vranceanu metrics and their Hopf bundles.
\end{abstract}

Keywords: Big tangent bundle; Courant bracket; (almost) Dirac structure; Lie integrability.

Mathematics Subject Classification 2010: 53C60, 53C05, 53C15, 53C20, 53C55, 53D18

Fix $M$ a smooth and connected $m \geq 2$-dimensional manifold with the tangent bundle $T M$ and cotangent bundle $T^{*} M$. The $C^{\infty}(M)$-module of sections of these bundles are: $\mathcal{X}(M)=\Gamma(T M)$ the Lie algebra of vector fields on $M$ and $\Omega^{1}(M)=$ $\Gamma\left(T^{*} M\right)$ the real linear space of 1 -forms on $M$.

The manifold $T^{\mathrm{big}} M:=T M \oplus T^{*} M$ is called big tangent bundle in [8] and the Pontryagin bundle of $M$ in [5]; for simplicity we will use the first name. This manifold is the total space of a vector bundle $\pi: T^{\text {big }} M \rightarrow T M$ with $\pi$ the first projection and $\Gamma\left(T^{\text {big }} M\right)=\mathcal{X}(M) \otimes \Omega^{1}(M)$ is endowed with the Courant structure $(\langle\rangle,,[]),,[2]:$

(1) the (neutral) inner product:

$$
\langle(X, \alpha),(Y, \beta)\rangle=\frac{1}{2}(\beta(X)+\alpha(Y)),
$$


(2) the (skew-symmetric) Courant bracket:

$$
[(X, \alpha),(Y, \beta)]=\left([X, Y], \mathcal{L}_{X} \beta-\mathcal{L}_{Y} \alpha-\frac{1}{2} d(\beta(X)-\alpha(Y))\right),
$$

where $\mathcal{L}_{X}$ is the Lie derivative with respect to the vector field $X \in \mathcal{X}(M)$.

Definition 1 ([2]). A vector sub-bundle $L$ of $T^{\text {big }} M$ which is $\langle$,$\rangle -isotropic and$ of maximal rank $m$ is called almost Dirac structure on $M$. If, in addition, the $C^{\infty}(M)$-module $\Gamma(L)$ of sections of $L$ is [, ]-integrable then $L$ is a Dirac structure.

Two associated notions are:

(1) a pair of complementary Dirac subspaces is called a reflector $([4,8])$ or Manin triple in [6],

(2) if $L$ is a Dirac structure on $M$ then the triple $\left(L,\left.[]\right|_{L},, p r: L \rightarrow T M\right)$ is a Lie algebroid on $M$ ([2, Theorem 2.3.4]).

Fix $F$ a vector sub-bundle of $T M$ and denote by $F^{0}$ its annihilator, that means the vector sub-bundle of $T^{*} M$ which vanish on $F$. Then $L_{F}=F \oplus F^{0}$ is a vector sub-bundle of rank $m$ of $T^{\mathrm{big}} M$.

The main result of this short note is a version of [8, Example 2.3].

Theorem 2. $L_{F}$ is an almost Dirac structure which is Dirac on $M$ if and only if $F$ is Lie integrable.

Proof. $L_{F}$ is maximally isotropic with respect to the inner product (1). Suppose now that $F$ is Lie integrable; then it defines a foliation $\mathcal{F}$ of $M$. The elements of $\Gamma\left(F^{0}\right)$ are 1 -forms which vanish by restriction to each leaf of $\mathcal{F}$. Also, their exterior derivative induce zero 2 -forms on the leaves of $\mathcal{F}$. Using these facts, it results that the Courant bracket of any two sections of $L_{F}$ is:

$$
[(X, \alpha),(Y, \beta)]=([X, Y], 0),
$$

which yields the conclusion about the Dirac structure of $L_{F}$.

Conversely, if $L_{F}$ is a Dirac structure from the Lie algebroid structure of $L_{F}$ we get that $\operatorname{pr}\left(L_{F}\right)$ is an integrable generalized distribution in the StefanSussman sense. But here $\operatorname{pr}\left(L_{F}\right)$ being exactly $F$ is a true vector sub-bundle of $T M$ i.e. of constant rank and then the Stefan-Sussman integrability is exactly the Lie-Frobenius one.

Example 3 (Ehresmann connection). Let $\xi=(M, \pi, B, F)$ be a fiber bundle; we use the approach of $\left[1\right.$, p. 105]. Let $n$ be the dimension of $B$ and $x=\left(x^{i} ; 1 \leq\right.$ $i \leq n)$, respectively $y=\left(y^{\alpha} ; 1 \leq \alpha \leq m-n\right)$ the coordinates on $B$ respectively $F$. Then, $(x, y)=\left(x^{i}, y^{\alpha}\right)$ are the coordinates on $M$. Applying the tangent functor to the bundle $\xi$ we get $T \pi: T M \rightarrow T B$ and then the kernel of $T \pi$ is a vector bundle over $M$ denoted $T_{V, \pi} M$ and called the vertical bundle of $\xi$. Its space of sections $\Gamma\left(T_{V, \pi} M\right)$ is spanned by $\left\{\frac{\partial}{\partial y^{\alpha}}\right\}$. 
Let an Ehresmann connection $E$ on $\xi$ with the horizontal vector sub-bundle $T_{H, \pi} M$ over $M$, complementary to $T_{V, \pi} M$ in $T M,[1$, p. 107]. It results the existence of smooth functions $\left\{N_{i}^{\alpha}(x, y)\right\}$ on $M$, called the coefficients of connection, such that $\Gamma\left(T_{H, \pi} M\right)$ is spanned by $\left\{\frac{\delta}{\delta x^{i}}=\frac{\partial}{\partial x^{i}}-N_{i}^{\alpha} \frac{\partial}{\partial y^{\alpha}}\right\}$.

Let $T_{V, \pi}^{*} M$ be the annihilator of $F_{1}=T_{V, \pi} M$; then $\Gamma\left(T_{V, \pi}^{*} M\right)$ is spanned by $\left\{d x^{i}\right\}$. Similarly, let $T_{H, \pi}^{*} M$ be the annihilator of $F_{2}=T_{H, \pi} M$; it results $\Gamma\left(T_{H, \pi}^{*} M\right)$ is spanned by $\left\{\delta y^{\alpha}=d y^{\alpha}+N_{i}^{\alpha} d x^{i}\right\}$. Our main objects in the following are the vector bundles over $M: L_{F_{1}}=V_{D}(\pi, E)=\left(T_{V, \pi} M, T_{V, \pi}^{*} M\right)$, respectively $L_{F_{2}}=$ $H_{D}(\pi, E)=\left(T_{H, \pi} M, T_{H, \pi}^{*} M\right)$. The rank of both is $m$.

We compute:

$$
\mathcal{L}_{\frac{\delta}{\delta x^{i}}} \delta y^{\alpha}=R_{i k}^{\alpha} d x^{k}-\frac{\partial N_{i}^{\alpha}}{\partial y^{\rho}} \delta y^{\rho}, \quad \mathcal{L}_{\frac{\partial}{\partial y^{\alpha}}} d x^{j}=0,
$$

where $R$ is the curvature of the Ehresmann connection $E$ :

$$
R_{i j}^{\alpha}=\frac{\delta N_{i}^{\alpha}}{\delta x^{j}}-\frac{\delta N_{j}^{\alpha}}{\delta x^{i}}
$$

provided by the condition of Lie integrability of the distribution $\Gamma\left(T_{H, \pi} M\right)$ :

$$
\left[\frac{\delta}{\delta x^{i}}, \frac{\delta}{\delta x^{j}}\right]=R_{i j}^{\alpha} \frac{\partial}{\partial y^{\alpha}} .
$$

Since $T_{V, \pi} M$ is integrable it results:

Corollary 4. $V_{D}(\pi, E)$ is a Dirac structure on $M . H_{D}(\pi, E)$ is an almost Dirac structure which is Dirac if and only if the connection $E$ is flat.

It results that a flat connection yields a Manin triple:

$$
T^{\mathrm{big}} M=V_{D}(\pi, E) \oplus H_{D}(\pi, E)
$$

in analogy with the decomposition of the tangent bundle provided by the Ehresmann connection:

$$
T M=T_{V, \pi} M \oplus T_{H, \pi} M
$$

From:

$$
\left\langle\left(\frac{\partial}{\partial y^{\alpha}}, d x^{i}\right),\left(\frac{\delta}{\delta x^{j}}, \delta y^{\beta}\right)\right\rangle=\frac{1}{2}\left(\delta_{j}^{i}+\delta_{\alpha}^{\beta}\right)
$$

we get that the decomposition (6) is not $\langle$,$\rangle -orthogonally. Therefore, we obtain$ two Lie algebroids over $M$ provided by a flat connection.

On this example we will treat other two main constructions of [2]. Firstly, let $\pi^{*}: T^{\mathrm{big}} T M \rightarrow T^{*} M$ be the canonical second projection. Relation $(2.2 .3-4)$ of $\left[2\right.$, p. 645] states that for a general almost Dirac structure we have: $\pi(L)=L \cap T^{*} M$ and $\pi^{*}(L)=(L \cap T M)^{0}$ and a skew-symmetric map $\Omega: \pi(L) \rightarrow \pi^{*}(L)$ is derived with the formula: $\Omega(\pi(u))=\left.\pi^{*}(u)\right|_{\pi(L)}$. In our framework we get: $\pi\left(L_{F}\right)=F$ and 
$\pi^{*}\left(L_{F}\right)=F^{0}$. Also, we have:

(1) $\Omega_{V}: T_{V, \pi} M \rightarrow T_{V, \pi}^{*} M$ is $\Omega\left(\left.\frac{\partial}{\partial y^{\alpha}}\right|_{(x, y)}\right)=\left.d x^{i}\right|_{(x, y)}$,

(2) $\Omega_{H}: T_{H, \pi} M \rightarrow T_{H, \pi}^{*} M$ is $\Omega\left(\left.\frac{\delta}{\delta x^{i}}\right|_{(x, y)}\right)=\left.\delta y^{\alpha}\right|_{(x, y)}$,

for every $(x, y) \in M$ since as it is pointed out several times in [2] in the manifold case we need to work pointwise.

Secondly, a function $f \in C^{\infty}(M)$ is called admissible $([2$, p. 650]) or Hamiltonian ([8]) if there exists a vector field $X_{f} \in \mathcal{X}(M)$ such that $\left(X_{f}, d f\right) \in \Gamma(L)$ and then $X_{f}$ is called the Hamiltonian vector field of $f$ and a bracket on admissible functions is given by $\{f, g\}=X_{f}(g)$. So, for $T_{V, \pi} M$ we get that the coordinate functions $x^{i}$ are admissible functions and the bracket is trivial:

$$
\left\{x^{i}, x^{j}\right\}=\frac{\partial x^{j}}{\partial y^{\alpha}}=0
$$

where $\frac{\partial}{\partial y^{\alpha}}$ is the Hamiltonian vector field of $f=x^{i}$ since $\left(\frac{\partial}{\partial y^{\alpha}}, d x^{i}\right) \in \Gamma\left(T_{V, \pi} M\right)$. $T_{H, \pi} M$ has $\left\{y^{\alpha}\right\}$ as admissible functions only if the Ehresmann connection has vanishing coefficients, i.e. $N_{i}^{\alpha}=0$, in which case $\frac{\delta}{\delta x^{i}}=\frac{\partial}{\partial x^{i}}$ are the corresponding Hamiltonian vector fields and again the bracket is trivial.

Particular Case 5 (Riemannian geometry). Suppose that $\xi$ is exactly the tangent bundle of $B$ i.e. $M=T B$. Then $m=2 n, F=\mathbb{R}^{n}$ and $\alpha=k$. Every Riemannian metric $g$ on $B$ gives rise to the Levi-Civita connection $E$ where $N_{i}^{k}=$ $\Gamma_{i a}^{k}(x) y^{a}$, with $\left(\Gamma_{i j}^{k}\right)$ being the Christoffel symbols of $g$. Then, a flat Riemannian metric on $B$ yields two complementary Dirac structures on $T B$.

Let us remark that some classes of Dirac structures naturally associated to Lagrangian systems appear in [11].

Remark 6 (The almost symplectic point of view). Let us recall that the big tangent bundle has also a non-degenerate skew-symmetric 2-form, [8]:

$$
\Omega((X, \alpha),(Y, \beta))=\frac{1}{2}(\beta(X)-\alpha(Y)) .
$$

From:

$$
\Omega\left(\left(\frac{\partial}{\partial y^{\alpha}}, d x^{i}\right),\left(\frac{\partial}{\partial y^{\beta}}, d x^{j}\right)\right)=0, \quad \Omega\left(\left(\frac{\delta}{\delta x^{i}}, \delta y^{\alpha}\right),\left(\frac{\delta}{\delta x^{j}}, \delta y^{\beta}\right)\right)=0
$$

we get that the complementary Dirac structures $V_{D}(\pi, E)$ and $H_{D}(\pi, E)$ are $\Omega$-Lagrangian sub-bundles of $T^{\mathrm{big}} M$.

Example 7. Let $H_{3}$ be the 3-dimensional Heisenberg space, [3]; recall that $H_{3}=$ $\left(\mathbb{R}^{3}, g\right)$ with the Riemannian metric:

$$
g=d x^{2}+d y^{2}+\left(d z+\frac{y}{2} d x-\frac{x}{2} d y\right)^{2} .
$$

An orthonormal basis of left-invariant vector fields on this Lie group is:

$$
X_{1}=\frac{\partial}{\partial x}-\frac{y}{2} \frac{\partial}{\partial z}, \quad X_{2}=\frac{\partial}{\partial y}+\frac{x}{2} \frac{\partial}{\partial z}, \quad X_{3}=\frac{\partial}{\partial z}
$$


with the dual frame:

$$
\theta_{1}=d x, \quad \theta_{2}=d y, \quad \theta_{3}=d z+\frac{y}{2} d x-\frac{x}{2} d y .
$$

Let $(x, y, z, X, Y, Z)$ be the coordinates on $T H_{3}$ and $\left(x, y, z, p_{x}, p_{y}, p_{z}\right)$ the coordinates on $T^{*} H_{3}$; then $T^{\text {big }} H_{3}$ has the coordinates $\left(x, y, z, X, Y, Z, p_{x}, p_{y}, p_{z}\right)$. Let the locally trivial fiber bundle $\pi: M=H_{3} \rightarrow B=\mathbb{R}^{2}, \pi(x, y, z)=(x, y)$, then $m=3$ and $n=2$. Fix the connection $E=E_{g}$ such that $T_{H, \pi} H_{3}$ is the $g$-orthogonal complement of $T_{V, \pi} H_{3}$.

Then $\Gamma\left(T_{V, \pi} H_{3}\right)$ is spanned by $X_{3}$ while $\Gamma\left(T_{V, \pi}^{*} H_{3}\right)$ is spanned by $\left\{\theta_{1}, \theta_{2}\right\}$; $\Gamma\left(T_{H, \pi} H_{3}\right)$ is spanned by $\left\{\frac{\delta}{\delta x}=X_{1}, \frac{\delta}{\delta y}=X_{2}\right\}$ and $\Gamma\left(T_{H, \pi}^{*} H_{3}\right)$ is spanned by $\left\{\theta_{3}\right\}$. We get the coefficients of the connection:

$$
N_{1}^{1}=\frac{y}{2}, \quad N_{2}^{1}=-\frac{x}{2}
$$

and therefore the curvature is:

$$
R_{12}^{1}=-R_{21}^{1}=1
$$

(Of course, we know that $g$ is not a flat metric but we include these computations in order to illustrate completely this example.) In conclusion, the only Dirac structure provided by Corollary 4 on $H_{3}$ is:

$$
V_{D}\left(\pi, E_{g}\right)=\left\{\left(x, y, z, 0,0, Z, p_{x}, p_{y}, 0\right) \in T^{\text {big }} H_{3}\right\}
$$

while:

$$
H_{D}\left(\pi, E_{g}\right)=\left\{\left(x, y, z, X-\frac{y}{2} Z, Y+\frac{x}{2} Z, 0,0,0, p_{z}+\frac{y}{2} p_{x}-\frac{x}{2} p_{y}\right) \in T^{\mathrm{big}} H_{3}\right\}
$$

is an almost Dirac structure on $\mathrm{H}_{3}$.

Example 8. Fix $k$ and $\tau$ two real numbers and denotes by $M_{k}^{3}$ the manifold $\left\{(x, y, z) \in \mathbb{R}^{3} ; F(x, y, z)=1+k\left(x^{2}+y^{2}\right)>0\right\}$. We shall consider on $M_{k}^{3}$ the Bianchi-Cartan-Vranceanu metric, [9, p. 343]:

$$
g_{k, \tau}=\frac{1}{F^{2}} d x^{2}+\frac{1}{F^{2}} d y^{2}+\left(d z+\frac{\tau y}{2 F} d x-\frac{\tau x}{2 F} d y\right)^{2} .
$$

For $k=0$ and $\tau=1$ we get the previous example; for other remarkable examples as well as the history of these metrics see [9].

An orthonormal basis in $\mathcal{X}\left(M_{k}^{3}\right)$ is:

$$
X_{1}=F \frac{\partial}{\partial x}-\frac{\tau y}{2} \frac{\partial}{\partial z}, \quad X_{2}=F \frac{\partial}{\partial y}+\frac{\tau x}{2} \frac{\partial}{\partial z}, \quad X_{3}=\frac{\partial}{\partial z}
$$

with the dual frame:

$$
\theta_{1}=\frac{d x}{F}, \quad \theta_{2}=\frac{d y}{F}, \quad \theta_{3}=d z+\frac{\tau y}{2 F} d x-\frac{\tau x}{2 F} d y .
$$

Let $M_{k}^{2}=\left\{(x, y) \in \mathbb{R}^{2} ; F(x, y)>0\right\}$ with the metric $g_{k}=\frac{d x^{2}+d y^{2}}{F^{2}}$. Then the map $\pi:\left(M_{k}^{3}, g_{k, \tau}\right) \rightarrow\left(M_{k}^{2}, g_{k}\right), \pi(x, y, z)=(x, y)$ being a Riemannian submersion yields 
a fiber bundle called Hopf bundle in [9, p. 344] since for $k=\frac{\tau^{2}}{4}$ we get the Hopf fibration $\pi: S^{3}(k) \rightarrow S^{2}(4 k)$. Again, let the connection $E=E_{g}$ such that $T_{H, \pi} M_{k}^{3}$ is the $g_{k, \tau}$-orthogonal complement of $T_{V, \pi} M_{k}^{3}$.

Then $\Gamma\left(T_{V, \pi} M_{k}^{3}\right)$ is spanned by $X_{3}$ while $\Gamma\left(T_{V, \pi}^{*} M_{k}^{3}\right)$ is spanned by $\{\delta x=$ $\left.F \theta_{1}, \delta y=F \theta_{2}\right\} ; \Gamma\left(T_{H, \pi} M_{k}^{3}\right)$ is spanned by $\left\{\frac{\delta}{\delta x}=\frac{1}{F} X_{1}, \frac{\delta}{\delta y}=\frac{1}{F} X_{2}\right\}$ and $\Gamma\left(T_{H, \pi}^{*} M_{k}^{3}\right)$ is spanned by $\left\{\theta_{3}\right\}$. We get the coefficients of the connection:

$$
N_{1}^{1}=\frac{\tau y}{2 F}, \quad N_{2}^{1}=-\frac{\tau x}{2 F}
$$

and therefore the curvature is:

$$
R_{12}^{1}=-R_{21}^{1}=\frac{\tau}{F^{2}}
$$

In conclusion, the only Dirac structure provided by Corollary 4 on $M_{k}^{3}$ is:

$$
V_{D}\left(\pi, E_{g}\right)=\left\{\left(x, y, z, 0,0, Z, p_{x}, p_{y}, 0\right) \in T^{\mathrm{big}} M_{k}^{3}\right\}
$$

while:

$$
H_{D}\left(\pi, E_{g}\right)=\left\{\left(x, y, z, X-\frac{\tau y}{2 F} Z, Y+\frac{\tau x}{2 F} Z, 0,0,0, p_{z}+\frac{\tau y}{2 F} p_{x}-\frac{\tau x}{2 F} p_{y}\right) \in T^{\mathrm{big}} M_{k}^{3}\right\}
$$

is an almost Dirac structure on $M_{k}^{3}$.

Remark 9 (A relationship with previous works). Returning to the general framework, let us recall that a connection on $\xi$ is equivalent with an almost product structure $P$ on $M$ such that $T_{V, \pi} M$ is the eigenbundle corresponding to the eigenvalue -1 of $P$ while $T_{H, \pi} M$ is the eigenbundle corresponding to the eigenvalue +1 of $P$ :

$$
P\left(\frac{\partial}{\partial x^{i}}\right)=\frac{\partial}{\partial x^{i}}-2 N_{i}^{\alpha} \frac{\partial}{\partial y^{\alpha}}, \quad P\left(\frac{\partial}{\partial y^{\alpha}}\right)=-\frac{\partial}{\partial y^{\alpha}}
$$

So, our Corollary 4 can be thought in correspondence with Example 4 from [10, p. 894]; the present result is more efficient than Proposition 2.3 of [10, p. 891] since one of the generated almost Dirac structure is in fact already Dirac. The flatness of the connection is equivalent with the integrability of $P$ expressed as the vanishing of the Nijenhuis tensor of $P$ and then we recover the Theorem 3.3 of [10, p. 892].

\section{References}

[1] A. M. Bloch, Nonholonomic Mechanics and Control, With the collaboration of J. Baillieul, P. Crouch and J. Marsden, With scientific input from P. S. Krishnaprasad, R. M. Murray and D. Zenkov. Interdisciplinary Applied Mathematics; Vol. 24, Systems and Control (Springer-Verlag, New York, 2003), MR1978379 (2004e:37099).

[2] T. J. Courant, Dirac manifolds, Trans. Amer. Math. Soc. 319(2) (1990) 631-661, MR0998124 (90m:58065).

[3] A. Ibort, T. Rodríguez de La Peña and R. Salmoni, Dirac structures and reduction of optimal control problems with symmetries, preprint (2010), arXiv: 1004.1438v1. 
[4] G. R. Jensen and M. Rigoli, Neutral surfaces in neutral four-spaces, Matematiche (Catania) 45(2) (1990) 407-443, MR1152942 (93d:53084).

[5] M. Jotz, T. S. Ratiu and J. Śniatycki, Singular reduction of Dirac structures, Trans. Amer. Math. Soc. 363 (2011) 2967-3013, MR2775795, http://arXiv.org/abs/ $0901.3062 \mathrm{v} 5$.

[6] Z.-J. Liu, A. Weinstein and P. Xu, Manin triples for Lie bialgebroids, J. Differential Geom. 45(3) (1997) 547-574, MR1472888 (98f:58203).

[7] H. J. Sussmann, Orbits of families of vector fields and integrability of distributions, Trans. Amer. Math. Soc. 180 (1973) 171-188, MR0321133 (47 \#9666).

[8] I. Vaisman, Isotropic subbundles of $T M \oplus T^{*} M$, Int. J. Geom. Meth. Mod. Phys. 4(3) (2007) 487-516, MR2343378 (2008g:53102).

[9] J. Van der Veken, Higher order parallel surfaces in Bianchi-Cartan-Vranceanu spaces, Results Math. 51(3-4) (2008) 339-359, MR2400172 (2009h:53128).

[10] A. Wade, Dirac structures and paracomplex manifolds, C. R. Math. Acad. Sci. Paris 338(11) (2004) 889-894, MR2059668 (2005a:53140).

[11] H. Yoshimura and J. E. Marsden, Dirac structures in Lagrangian mechanics. I, II, J. Geom. Phys. 57(1) (2006) 133-156, 209-250, MR2265464 (2007j:37087), MR2265469 (2007j:37088). 